\title{
TRPA1 deficiency reduces skin inflammation in 2,4-dinirtochlorobenzene-induced atopic dermatitis animal model
}

\author{
Dan Zeng ${ }^{1}$, Chao Chen ${ }^{1}$, Wei Zhou ${ }^{1}$, Xuesu $\mathrm{Ma}^{1}$, Xi $\mathrm{Pu}^{1}$, Yue Zeng ${ }^{1}$, Fenglin $\mathrm{Lv}^{2}$, and \\ Weikang Zhou ${ }^{1}$ \\ ${ }^{1}$ Department of AllergyChongqing General HospitalUniversity of Chinese Academy of \\ Sciences \\ ${ }^{2}$ College of Bioengineering, "111 Project" Laboratory of Biomechanics and Tissue Repair \\ Engineering, Key Laboratory of Biorheological Science and Technology, Chongqing \\ University
}

May 11, 2020

\begin{abstract}
Background: Atopic dermatitis (AD) is a chronic inflammatory skin disorder characterized by severe itching and recurrent eczema-like lesions. Yet, its exact pathological mechanism remains unclear. Objective: The aim of this study was to investigate the role of TRPA1 in the pathogenesis of AD. Methods: The experimental atopic dermatitis (AD)-like skin lesions were established using 2,4-dinitrochlorobenzene (DNCB). Mice were divided into three groups: TRPA1-/- and WT groups were treated with DNCB dissolved in a 3:1 mixture of acetone and olive oil and the negative control group was treated with 3:1 mixture of acetone and olive oil without DNCB. The treatment lasted for 21 days, after which the animals were sacrificed and their blood, ears and dorsal skin tissue samples were collected for analysis. Results: Lower dermatitis score, ear thickness, pruritus score, and epidermal hyperplasia were observed in mice in TRPA1-/- mice compared to the WT group. Besides, lower dermal mast cell infiltration, proinflammatory cytokines, Th2 cytokines and the infiltration of macrophages were observed in the TRPA1-/- mice compared to the WT group. Furthermore, we demonstrated that TRPA1 antagonist HC-030031 could alleviate AD-like symptoms and reduce the degree of epidermal hyperplasia in mice. Conclusions: TRPA1 has a crucial role during the $\mathrm{AD}$ pathogenesis in mice, thus could be used as a potential new target for treating patients with chronic skin inflammatory disease.
\end{abstract}

\section{Introduction}

Atopic dermatitis (AD) is a chronic inflammatory skin disease with typical symptoms like severe itching and recurrent eczema-like lesions [1], which affects $5 \%$ to $20 \%$ of children and $2 \%$ to $10 \%$ of adults worldwide $[2,3]$. The pathophysiology of $\mathrm{AD}$ may involve a complex interaction between genetic and environmental factors that are associated with immunity, skin barrier dysfunction, pruritus and other factors [4]. Although there is no cure for $\mathrm{AD}$, treatment can decrease dryness and irritation. Steroids or calcineurin inhibitors are the most commonly used medication. Yet, those drugs may not always be effective and may induce side effects, especially if used in children over a longer time [5]. Thus, there is an urgent need to further explore the exact mechanism of pathogenesis in order to find effective therapeutic targets for the treatment of AD.

TRPA1 is a six-time transmembrane protein that belongs to the TRP channel family, which is activated under some environmental stimuli, such as low temperature, acidic environment and hydrogen peroxide rich environment $[6,7]$. TRPA1 is expressed in peripheral sensory nerve fibers, but also a variety of cells, such as cutaneous keratinocytes, mast cells, endothelial and dendritic cells. It is also involved in inflammation, neuropathic pain and is a key downstream signal of histamine-independent pruritus [8,9]. Recent evidence 
suggests that TRPA1 has a vital role in pruritus. Studies have shown that hapten oxazolidone (Oxa) and squaric acid dibutylester (SADBE) can directly activate the TRPA1 channel and participate in inducing itching in allergic contact dermatitis. For example, Oxa-induced skin inflammation has been alleviated in TRPA1 deficiency mice while SADBE-induced skin inflammation remains [10, 11]. Moreover, Imiquimod (IMQ) can directly activate the TRPA1 channel and has an inflammatory protective role in psoriasiform dermatitis $[12,13]$. Recent studies have found that TRPA1 is highly expressed in skin lesions of AD patients with severe pruritus [14]. Although the role of TRPA1 in AD pruritus has been widely reported, the effect of TRPA1 in the pathogenesis of $\mathrm{AD}$, especially in the regulation of inflammation of $\mathrm{AD}$ and the therapeutic effect of anti-TRPA1 in the treatment of $\mathrm{AD}$, remains unknown.

DNCB, a hapten that can activate the TRPA1 channel of HEK cells [15,16], can be used to further explore the role of TRPA1 in the pathogenesis of AD. The aim of this study was to investigate the role of TRPA1 in the pathogenesis of DNCB-induced $\mathrm{AD}$ in mice.

\section{Materials and methods}

\subsection{Animals}

TRPA1 ${ }^{-/}$mice were purchased from the Jackson Laboratories (Bar Harbor, ME, USA), while the C57/BL6 mice were purchased from the Animal Centre of Chongqing Medical University (Chongqing, China). All the animals were housed in an environment with a temperature of $22 \pm 1{ }^{\circ} \mathrm{C}$, relative humidity of $50 \pm 1 \%$, and a light/dark cycle of $12 / 12 \mathrm{hr}$. All animal studies (including the mice euthanasia procedure) were done in compliance with the regulations and guidelines of Chongqing medical University institutional animal care and were conducted according to the AAALAC and the IACUC guidelines. All mice in all the experiments were 12 -weeks old (20-25g).

\subsection{Induction of Topical AD-Like Skin Dermatitis in Mice by 2,4-dinitrochlorobenzene (DNCB)}

The experimental atopic dermatitis (AD)-like skin lesions were established by using 2,4-dinitrochlorobenzene (DNCB) according to the literature [17] with slight modification.

Mice were divided into three groups (6 mice/group): TRPA1 ${ }^{-/-}$and WT groups were treated with DNCB; the negative control group was treated with 3:1 mixture of acetone and olive oil without DNCB. Briefly, after 1 week of acclimation, the dorsal hair of mice was completely shaved with a clipper before sensitization. For the sensitization process, $150 \mu \mathrm{l}$ of $2 \%$ DNCB dissolved in a 3:1 mixture of acetone and olive oil was applied to the dorsal skin, and $10 \mu \mathrm{l}$ per ear was applied to both ears. After four days, $0.5 \%$ DNCB was applied to challenge the dorsal skin $(150 \mu \mathrm{l})$ and both ears $(10 \mu \mathrm{l}$ per ear) at the interval of two days for 20 days.

The treatment lasted for 21 days, after which the animals were sacrificed and their blood, ears and dorsal skin tissue samples were collected for analysis.

\subsection{Evaluation of dermatitis, ear thickness and scratching behavior}

The severity of dorsal skin lesions was evaluated based on four symptoms: edema, erythema/hemorrhage, scarring/dryness, and excoriation/erosion. A total dermatitis score was defined as the sum of the scores of individual symptoms ( 0 , no symptoms; 1 , mild symptoms; 2 , moderate symptoms; 3 , severe symptoms) ranging from 0 to 12 .

The ear thickness of the mouse was measured by an electronic caliper (Shanghai, China) with $0.1 \mathrm{~mm}$ accuracy. The scratching frequencies were measured according to the previously described criteria [13] with some modification. The mice were acclimated for $1 \mathrm{~h}$ by being individually placed into a recording cage, after which D NCB was applied to their dorsal skin and ears. After 30 minutes, the number of scratching bouts at the DNCB site was measured and recorded for 10 minutes.

\subsection{Histological and immunochemistry analysis}

The ear and dorsal skin were fixed with $4 \%$ paraformaldehyde, embedded in paraffin and cut into $5 \mu \mathrm{m}$ thick sections. Samples were then treated with Hematoxylin and Eosin (H\&E) to detect epidermal thickening 
and inflammatory infiltration. Toluidine blue (TB) was used to evaluate the infiltration of mast cells. The number of mast cells in five randomly targeted sites was counted using a light microscope (Nikon Eclipse $\mathrm{Ni}$, Tokyo, Japan) with $\times 200$ magnification.

\subsection{Serum IgE Measurements}

Blood samples were collected on day 21 and stored at - 80 degC. The level of $\operatorname{IgE}$ in the serum was quantified using a commercial enzyme-linked immunosorbent assay (ELISA) kit (4A Biotech, Beijing, China) according to the manufacturer's instructions.

\subsection{RNA isolation and quantitative RT-PCR analysis}

Complete RNA was extracted from dorsal skin tissue using TRIzol (TransGen Biotech, Beijing, China) according to the manufacturer's instructions. First-strand cDNA was synthesized using a RevertAid First Strand cDNA Synthesis Kit (ThermoScientific, MA, USA) following the manufacturer's instructions. Quantitative RT-PCR analysis of gene expression was done using FastStart Universal SYBR Green Master (Roche, Basel, Switzerland). The levels of different target genes normalized against GAPDH were calculated using $2-\Delta \Delta \mathrm{Ct}$ methods. Primer sequences (5' to $3^{\prime}$ ) were as follows: murine GAPDH forward: CTCGTCCCGTAGACAAAATGGT, reverse, GAGGTCAATGAAGGGGTCGTT; murine IL-1 $\beta$ forward: GAAATGCCACCTTTTGACAGTG, reverse, TGGATGCTCTCATCAGGACAG; murine IFN$\alpha$ forward: GATGCCCTGCTGGCTGTGA, reverse, CTTCTGCTCTGACCACCTCCC; murine IL-6 forward: CTGCAAGAGACTTCCATCCAG, reverse, AGTGGTATAGACAGGTCTGTTGG; murine IFN- $\gamma$ forward: TCGGTAACTGACTTGAATGTCCA, reverse, TCGCTTCCCTGTTTTAGCTGC; murine Il4 forward: GGTCTCAACCCCCAGCTAGT, reverse, GCCGATGATCTCTCTCAAGTGAT; murine Il13 forward: TGAGCAACATCACACAAGACC, reverse, GGCCTTGCGGTTACAGAGG.

\subsection{Protein extraction and western blot analysis}

Dorsal skin samples were dissected and stored at $-80{ }^{\circ} \mathrm{C}$. Protein lysates were prepared using lysis buffer containing a protease inhibitor, calcineurin inhibitors and PMSF. The concentration of protein in the lysates was measured using the BCA Protein Assay Kit (Beyotime, Shanghai, China) and equal amounts of protein were applied on 10\% SDS-PAGE gels. Separated proteins were electrophoretically transferred to PVDF membranes. After being blocked with 5\% skim milk, the membranes were incubated with anti-F4/80, CD206, iNOS (Abcam, Cambridge, UK; 1:1000) at 4 overnight. After washing, membranes were incubated with HRP-conjugated secondary antibodies at room temperature for $1 \mathrm{~h}$. Immunoreactive signals were detected using an enhanced chemiluminescence solution (ThermoScientific, MA, USA).

\subsection{Fluorescent immunohistochemistry analysis}

Immunofluorescent staining was performed using 4-um paraffin sections. Primary antibodies of F4/80; CD206; iNOS (Abcam, Cambridge, MA; 1:100), and corresponding secondary antibodies (Invitrogen, Carlsbad, CA, USA) were used for staining. Nuclei were stained with DAPI. The images were obtained by a laser scanning microscope system (Nikon Eclipse Ni, Tokyo, Japan).

\subsection{Statistical analysis}

Statistical analysis was performed using GraphPad Prism software (ver. 7.0 GraphPad Software, San Diego, CA, USA). Results are expressed as mean +- standard error of the mean(S.E.M). Student'st- test was used for comparisons between two groups. One-way ANOVA analyzed differences among multiple groups. $p$ values $<0.05$ were considered to be statistically significant.

\section{Results}

3.1 TRPA1 deficiency ameliorates skin inflammation and suppresses scratching behavior ina DNCB-induced AD mouse model

To investigate the significance of the TRPA1 in AD, repeated cutaneous allergen exposure with DNCB was used to induce the AD murine model as mentioned in Figure 1. Following DNCB treatment, the 
lesion skin of ears and dorsal with edema, erythema, excoriation, and scarring were sequentially observed. Interestingly, the dermatitis score was significantly decreased in DNCB-treated TRPA1 ${ }^{-/}$mice compared to DNCB-treated WT mice (Figure 2A ). Furthermore, we found that a TRPA1 ${ }^{-/}$had a protective effect in suppressing DNCB-induced ear thickening (Figure 2B ).

Consequently, we investigated the effects of TRPA1 on the scratching behavior in the DNCB-induced AD mouse model. The DNCB-treated WT mice showed more frequent scratching behavior compared to DNCBtreated TRPA1 ${ }^{-/-}$mice (Figure 2C ). Moreover, typical characteristics of AD histopathology, including marked epidermal hyperplasia and increased cellular were observed in DNCB-treated WT mice and DNCBtreated TRPA $1^{-/}$mice, as confirmed by microscopic analysis of $\mathrm{H} \& \mathrm{E}$ stained sections of dorsal and ear lesion skin (Figure 2D-2E ). Yet, the thickening of the epidermis and the infiltration of inflammatory cells were significantly decreased in DNCB-treated TRPA1 ${ }^{-/-}$mice than DNCB-treated WT mice (Figure 2F-2G ). These results suggested that TRPA1 deficiency not only alleviates pruritus, but also has an inflammatory protective role in $\mathrm{AD}$.

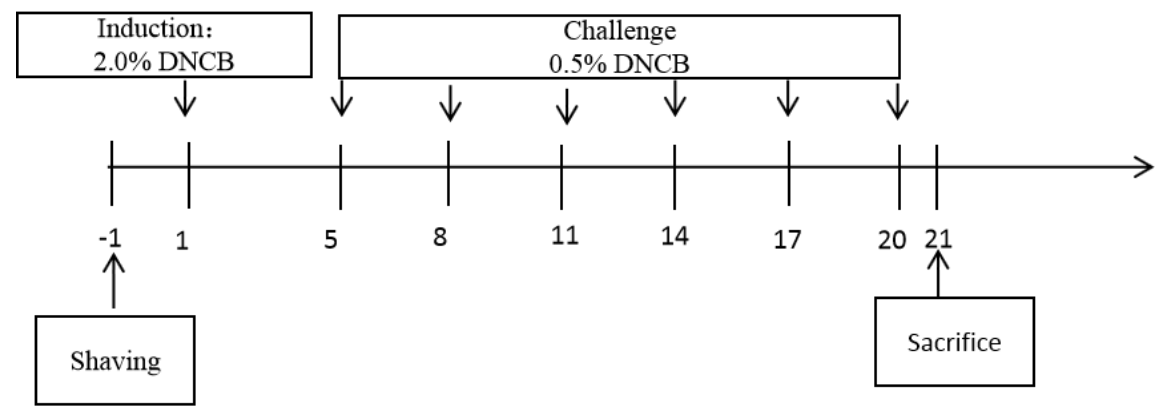

Fig.1 Schematic protocol of DNCB-induced AD model.

A

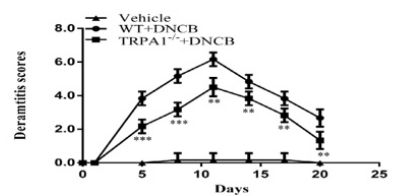

B

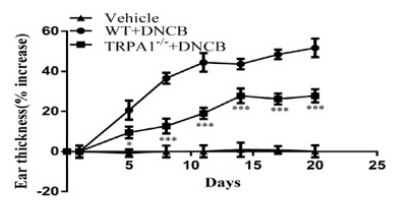

C

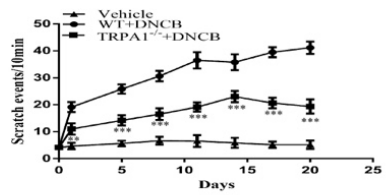

D Vehicle

WT+DNCB

TRPA1-/-+DNCB

E

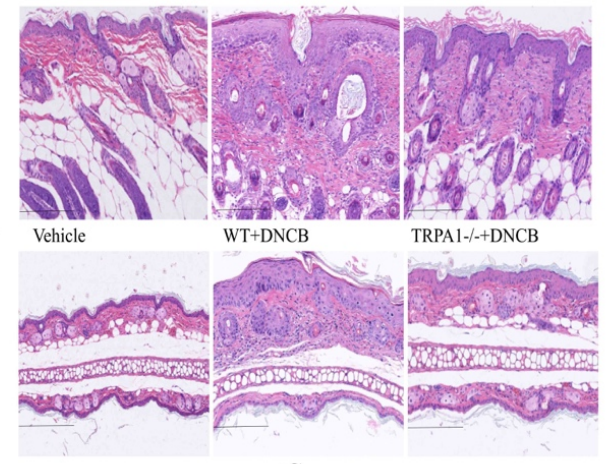

F

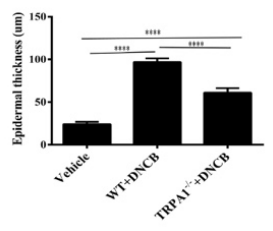

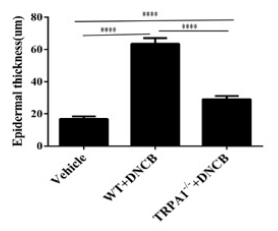

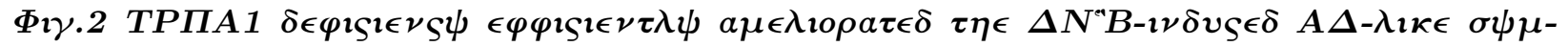

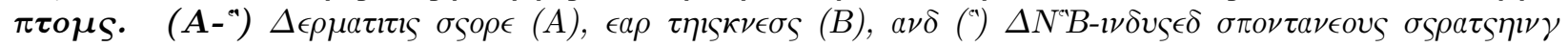

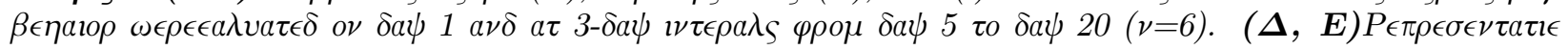




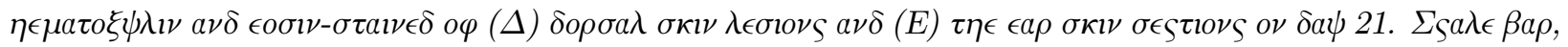

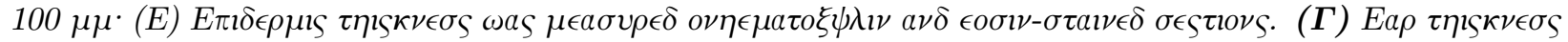

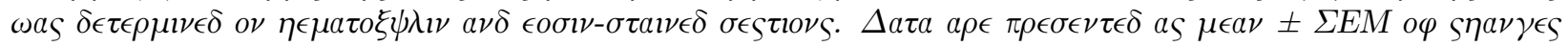
$\imath$ a $a \lambda \in \epsilon,{ }^{*} \pi<0.05,{ }^{* *} \pi<0.01$

\subsection{TRPA1 deficiency reduces mast cell infiltration and serum tIgE in DNCB-induced AD mouse model}

Mast cell infiltration and hyperproduction of IgE are the main features of AD. In this study, we used toluidine blue to stain the mast cells from dorsal and ear tissue sections. The results showed higher dermal mast cell numbers in DNCB-treated WT mice compared to DNCB-treated TRPA1 ${ }^{-/}$mice (Figure 3A-3D ). Besides, we assessed the levels of total IgE in the serum from mice collected on the last day of the experiment. The results showed that the level of serum total $\mathrm{IgE}$ in the serum was significantly increased by repeated DNCB stimulation in dorsal and ear; yet, no significant difference was observed between groups (Figure $\mathbf{4 E}$ ).
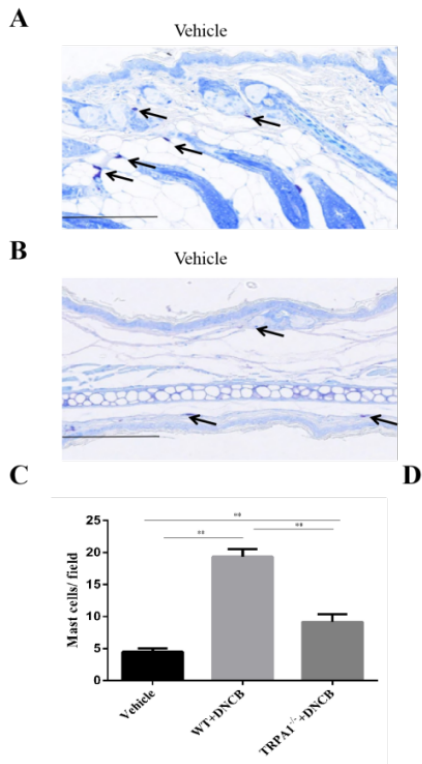

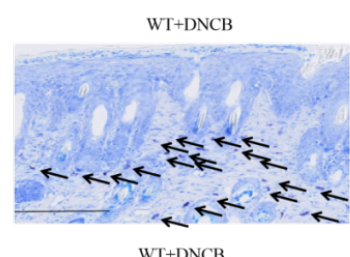

WT + DNCB
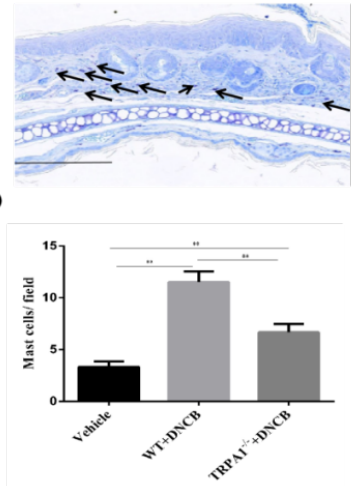

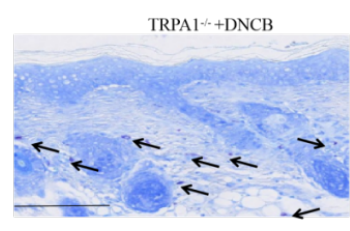

TRPAl $*+$ DNCB

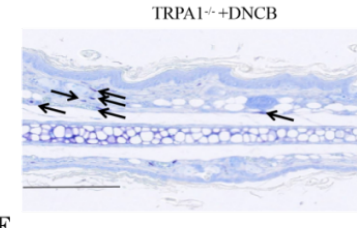

$\mathbf{E}$

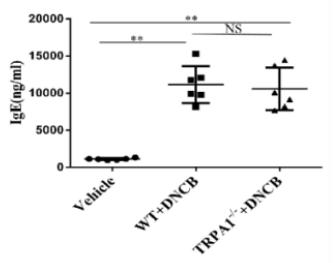

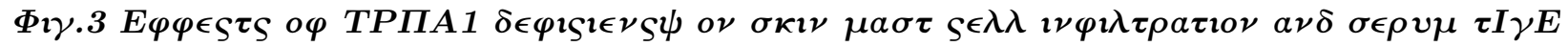

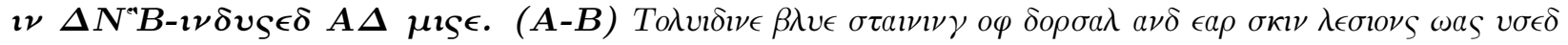

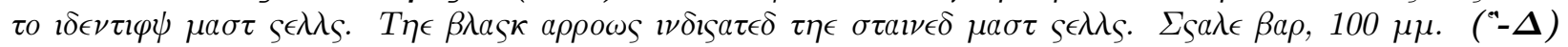

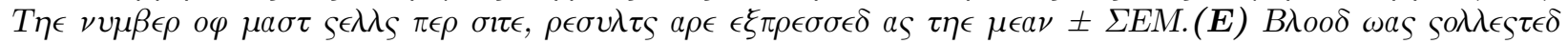

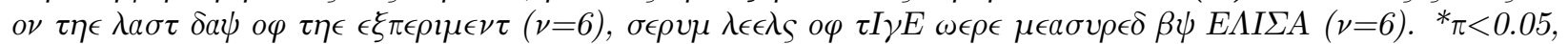
${ }^{* *} \pi<0.01, N \Sigma, \nu 0 \tau$ ol $\gamma \nu \varphi \imath \varsigma a \nu \tau$

3.3 TRPA1 regulates the expression of proinflammatory cytokines and Th2 cytokines in DNCB-induced AD mouse model

Next, we examined the mRNA expression of inflammatory cytokines in the dorsal skin of TRPA1 ${ }^{-/}$mice and WT mice treated with DNCB. As shown in Figure 4A-F, the DNCB induced a remarkable increase in the mRNA expression of IL-1 $\beta$, TNF- $\alpha$, IL-6, IL-4, IL-13. Most importantly, the expression of the proinflammatory cytokine, IL-1 $\beta$, TNF- $\alpha$, and IL- 6 in the skin lesions significantly decreased in DNCBtreated TRPA1 ${ }^{-/-}$mice compared to DNCB-treated WT mice (Figure $\mathbf{4 A - 4 C}$ ). Besides, we found that Th2 cytokine, IL-4, and IL-13 production were significantly reduced in DNCB-treated TRPA1 ${ }^{-/-}$mice compared to the WT mice (Figure 4E-F ); nevertheless, no difference in mRNA expression of IFN- $\gamma$ was found between groups (Figure 4D ). To sum up, the trends of cytokines expression might further explain the 
amelioration of DNCB-induced AD-like symptoms and skin pathology in TRPA1 /- mice.

A

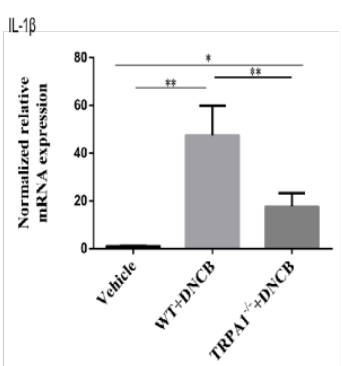

D

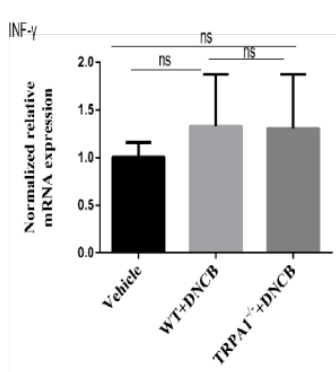

B

TNF:-0

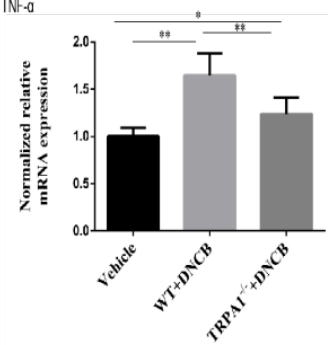

E

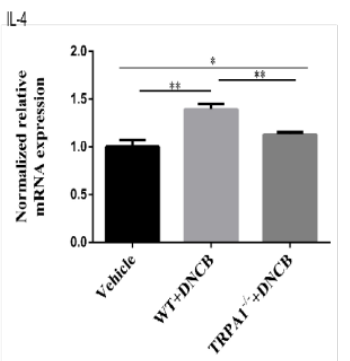

$\mathrm{C}$

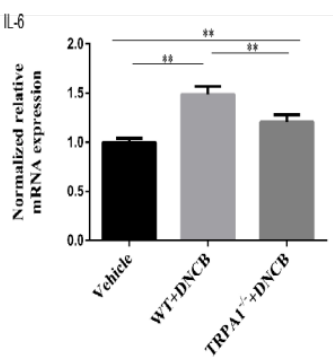

F

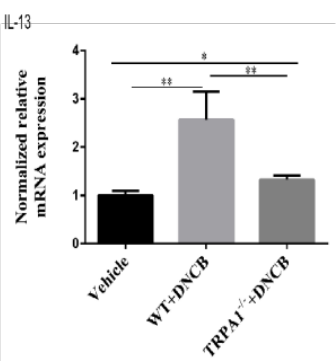

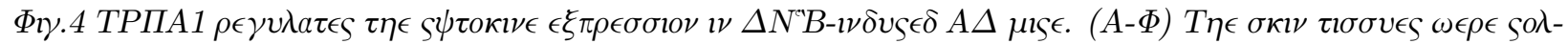

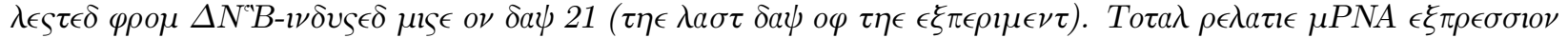

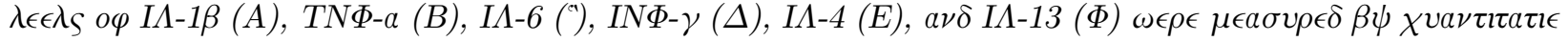

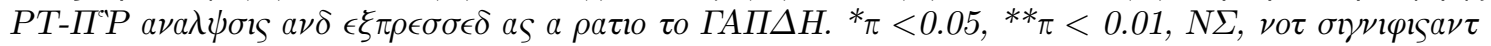

\subsection{TRPA1 deficiency inhibits DNCB-induced infiltration of macrophages in AD mice}

In chronic $\mathrm{AD}$, the increase of macrophage infiltration is closely related to the degree of skin damage and immune dysfunction. To verify the effect of TRPA1 on macrophage infiltration in DNCB-induced AD, the expression of F4/80 in the back skin was analyzed by immunofluorescence and Western blot. The results showed that the number of $\mathrm{F} 4 / 80+$ cells and its protein expression significantly increased after repeated skin stimulation with DNCB. Compared with the WT group, the number of $\mathrm{F} 4 / 80^{+}$cells and its protein expression in TRPA1-/- group decreased (Figure 5A-5B ).

There are two main pathways for macrophage activation, the classical M1 pathway and the alternative M2 pathway. In this study, we explored the effect of TRPA1 on the infiltration of M1 and M2 macrophages. The results showed that the expression of $\mathrm{iNOS}^{+}$and CD206 protein induced by DNCB were significantly increased and in the TRPA1-/- group, the expression of iNOS $^{+}$and CD206 protein decreased compared with the WT group (Figure 5A ). These results suggest that TRPA1 deficiency inhibits the infiltration of M1 and M2 macrophages in the lesions. 


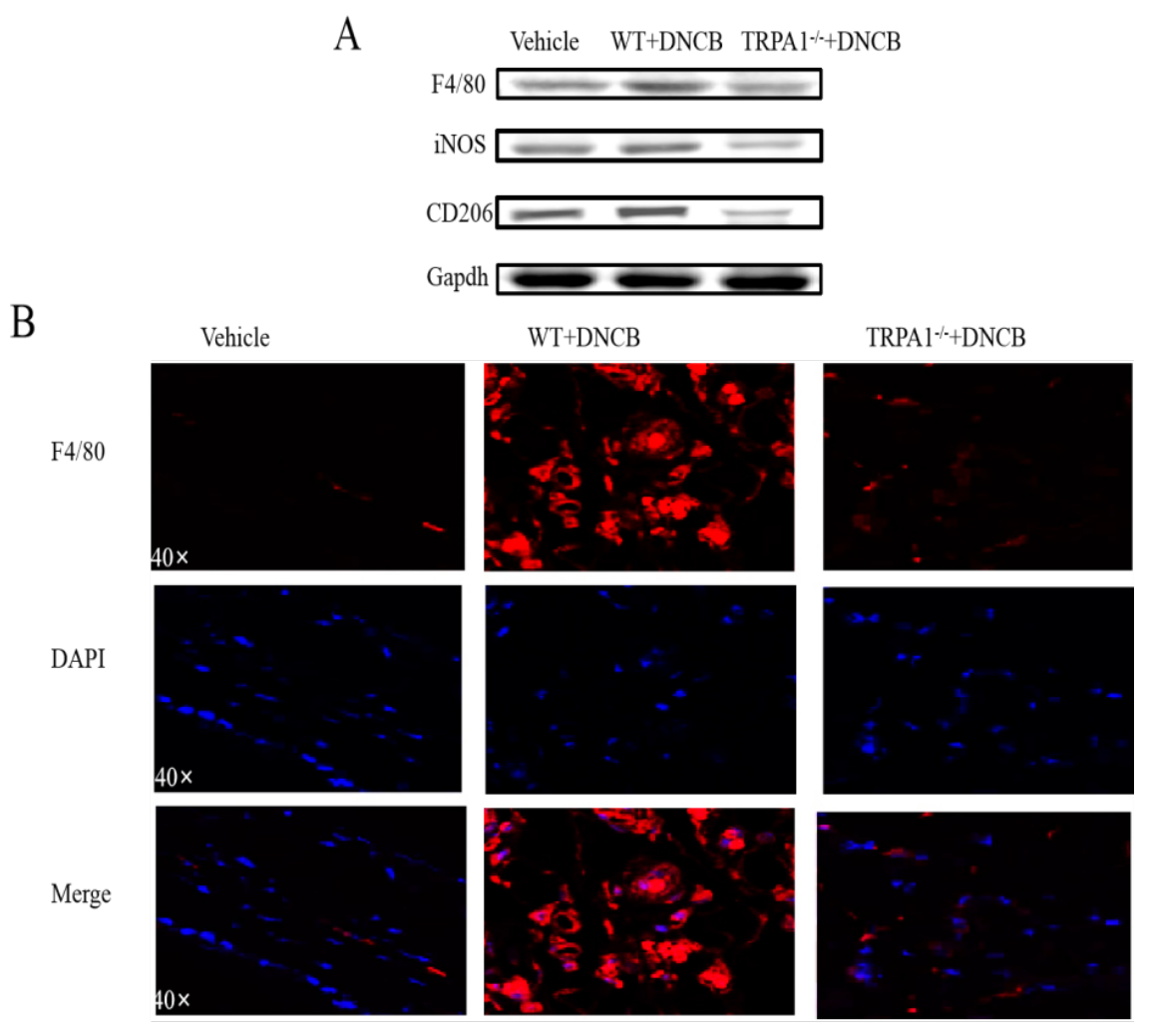

Fig.5 TRPA1 deficiency reduces macrophage infiltration in lesions. (A) The expression of F4/80, iNOS, CD206 and Gapdh using Western blot. (B) Immunofluorescence staining images of F4/80 (red) and DAPI (blue; $\times 40$.

\subsection{Administration TRPA1 antagonists HC030031 mitigates skin inflammation andscratching behavior in DNCB-induced AD mouse model}

To eliminate the possibility of compensatory mechanisms in the $\mathrm{KO}$ animals and explore whether selective TRPA1 antagonists HC030031 can be used as a targeted therapy for DNCB-induced AD. Experiments were carried out involving pre-treatment with the HC030031. Wild-type (WT) mice received an intraperitoneal injection of HC-030031 (TRPA1 receptor analog, 100mg/kg, ApexBio, USA). Applying HC-030031 prior to DNCB challenge alleviated atopic symptoms, ear thickness and scratching behavior (Figure 6A-6C ). At the same time, HC-030031 recovered histological abnormalities (Figure 6D-6E). Our experiments with TRPA1 antagonists were similar to the observed profile in TRPA1 KO mice. Altogether, the obtained results show that anti-TRPA1 represents a potential therapeutic target for AD disease. 
A

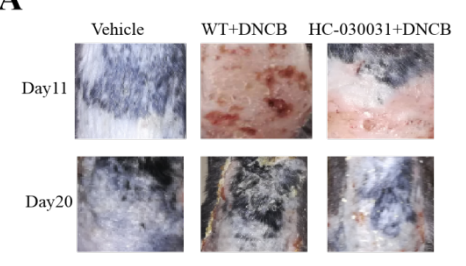

D

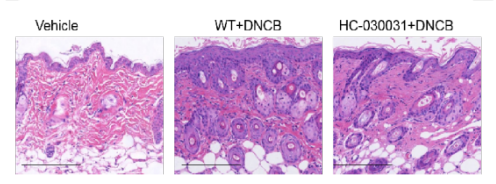

B

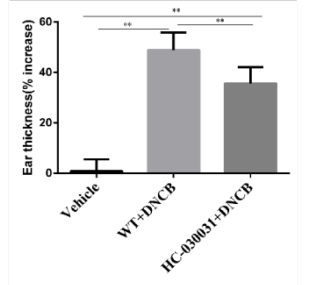

$\mathbf{E}$

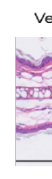

C

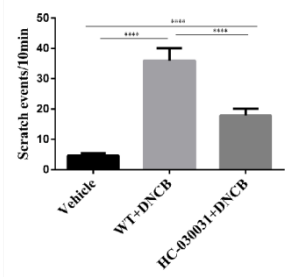

HC-030031+DNCB

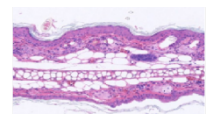

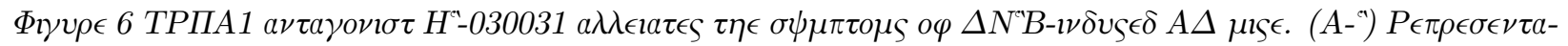

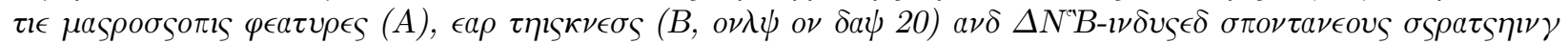

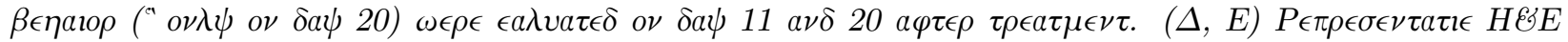

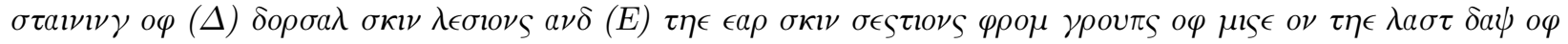
$\tau \eta \epsilon \epsilon \xi \pi \epsilon \rho \mu \epsilon \nu \tau(\delta a \psi 21) \Sigma_{\varsigma} a \lambda \epsilon \beta a \rho, 100 \mu \mu \cdot{ }^{*} \pi<0.05,{ }^{* *} \pi<0.01$

\section{Discussion}

A better understanding of the molecular and cellular mechanisms underlying AD is critical for developing new effective, and safe therapeutic strategies to treat the debilitating skin conditions. In this study, we investigated the anti-AD potential of TRPA1 by using TRPA1 knockout mice. Our data suggested that the genetic ablation of the TRPA1 could reduce the clinical features induced by DNCB in mice.

$\mathrm{AD}$ is a chronic inflammatory skin disease mainly characterized by itching symptoms (observed in $87 \%$ $100 \%$ patients) $[18,19]$. The vicious cycle of pruritus-scratch induces/aggravates inflammation of AD, and causes insomnia and fatigue, which seriously affects the patients' quality of life [20]. TRPA1 is the only member of the TRP channel anchor protein (TRPA) subfamily, which is widely expressed in sensory neurons and non-neuronal cells. TRPA1 is involved in the transmission of several different sensory modes (cold, pain, and pruritus) and in the mediation of neurogenic inflammation [21]. Current studies have shown that TRPA1 expression is up-regulated in AD patients, where it has an important role in histamine-independent pruritus. TSLP, an inflammatory cytokine, activates TRPA1 by binding it to the receptor TSLPR on sensory nerve fibers, causing pruritus in mice. Besides, TRPA1 has an essential role in Th2 cell-dependent pruritus mediated by IL-31 receptor expressed on sensory nerves. In the transgenic AD mice model with overexpression of IL-13, TRPA1 antagonists can significantly alleviate itching [22-25].

In the present study, we found that TRPA1 knockout reduces the frequency of itching symptoms and the severity of skin lesions in AD mice. Besides, the TRPA1 knockout alleviated the pathological change associated with AD by reducing the number of mast cells, serum IgE, inflammatory cytokines, and macrophage infiltration, which further suggests that TRPA1 has an inflammatory positive role in DNCB-induced AD. Hapten oxazolidone can activate the TRPA1 pathway, up-regulate inflammatory cytokines, neuropeptide (CXCL-2), and nerve growth factor (NGF) in chronic dermatitis induced by hapten oxazolidone and can promote the occurrence of inflammation and pruritus [11]. However, in allergic contact dermatitis induced by squaric acid dibutylester (SADBE), TRPA1 participates in chronic pruritus induced by SADBE, while epithelial skin inflammation persists in TRPA1 knockout mice [12]. We speculated that although TRPA1 participates in chronic pruritus induced by different hapten, its role in skin inflammation may be different. Our findings suggest that TRPA1 promotes DNCB-induced AD inflammation, which is consistent with the observation that TRPA1 knockout could reduce inflammatory cell infiltration and epidermal thickening.

Mast cells have an essential role in the pathogenesis of AD. Those cells can produce and secrete inflammatory mediators such as histamine, growth factors, cytokines, and chemokines [26]. A previous study [25] reported a 
high expression of TRPA1 in dermal afferent nerves and mast cells from patients with AD, but not in the skin from healthy subjects. Besides, the same study found that allyl isothiocyanate, TRPA1 agonist, increases the calcium signal of those cells [25]. Furthermore, Hoxet al found that TRPA1-dependent activation of sensory neurons and mast cells regulate the non-allergic airway hyperresponsiveness in mice [27]. In this study, we found a lower number of mast cells in DNCB treated- TRPA1-/- mice compared to DNCB treatedWT group. TRPA1 deficiency inhibited the infiltration of mast cells in the dermis of the DNCB-induced AD model, thus suggesting that TRPA1 may promote AD symptoms by promoting mast cell infiltration.

Immunological abnormalities of $\mathrm{AD}$ are characterized by the imbalance of Th1/Th2 and abnormal secretion of inflammatory factors. Previous studies have shown that toluene diisocyanate can activate the TRPA1 channel, while the antagonize TRPA1 can inhibit Th2 inflammation in allergic airway inflammation induced by TRPA1 [28]. Oxazolone can activate the TRPA1 channel, and TRPA1 can up-regulate pre-inflammation and Th2 related cytokines [11]. Our data indicated that TRPA1-/- reduces the expression of pro-inflammatory factors and Th2 inflammatory cytokines in DNCB-induced AD mice, thus suggesting that TRPA1 may promote the symptoms of $\mathrm{AD}$ by regulating pro-inflammatory factors (IL-1beta; IL-6), and Th-2 inflammatory cytokines (IL-4; IL-13).

Activation and sensitization of TRPA1 can regulate skin inflammation by increasing the release of inflammatory mediators; yet, the role of TRPA1 in immune cells needs to be further explored. In chronic AD, the increase of macrophage infiltration is closely related to the degree of skin damage and immune dysfunction [29]. Macrophages, which have a key role in the immune response, can be divided into two functional subgroups: macrophages polarized to M1 under the stimulation of interferon-gamma and lipopolysaccharide; and macrophages polarized to M2 under the stimulation of IL-4 and IL-13 [30]. F4/80 antigen is a mature mouse cell surface glycoprotein that is highly expressed in both M1 and M2 macrophages [31]. Our study showed that TRPA1 deficiency reduced the expression of F4/80 protein and the number of positive cells, and the expression of iNOS (expressed only M1 macrophages) and CD206 protein (expressed only M2 macrophages), thus suggesting that TRPA1 deficiency may inhibit the infiltration of dermal macrophages in DNCB-induced AD model. In addition, our data suggest that TRPA1 may promote AD inflammation by promoting macrophage infiltration. Although TRPA1 activator cinnamaldehyde inhibits the release of IL-1 $\beta$, IL-6 and TNF- $\alpha$ mediated by lipopolysaccharide and lipophosphatidic heterocyclic acid from mouse macrophages and human monocytes, it suggests that TRPA1 may have anti-inflammatory effects in monocytes $[9,32]$. However, in the DNCB-induced AD mice model, TRPA1 deficiency inhibits the infiltration of macrophages, which is consistent with the fact that TRPA1 mediates the infiltration of macrophages during corneal wound healing and antagonizing TRPA1 reduces the proportion of M2 macrophages to improve myocardial fibrosis $[33,34]$. It is also consistent with the decreased expression of inflammatory factors (IL- $1 \beta$. TNF- $\alpha$ IL-6) observed in our study. Yet, the role of TRPA1 in macrophages needs to be further elucidated.

Nowadays, the small molecule targeted therapy of AD has been attracting increasing attention [35]. We further explored the therapeutic effect of TRPA1 antagonist HC-030031 on the DNCB-induced AD model. Our results showed that HC-030031 can effectively alleviate skin lesions and pruritus in the AD mouse model. Pathological findings showed that it can alleviate inflammatory cell infiltration and epidermal thickening, similar to those observed in TRPA1 deficient mice. At the same time, the results showed that the TRPA1 antagonist had an anti-AD effect in the DNCB-induced AD model and could be used as one of the therapeutic targets of $\mathrm{AD}$.

In conclusion, our study demonstrated that TRPA1 has a crucial role during AD pathogenesis in mice. TRPA1 deficiency alleviated pruritus and showed an immunoprotective effect on DNCB-induced AD mice. In addition, TRPA1 antagonist HC-030031 had a certain therapeutic effect on DNCB-induced AD mice. These data suggest that TRPA1 can be used as a potential new target for treating patients with AD.

\section{References}

[1] Weidinger S, Novak N. Atopic dermatitis. Lancet 2016; 387(10023):1109-1122.

[2] Williams H, Stewart A, von Mutius E, Cookson W, Anderson H. Is eczema really on the increase world- 
wide? J Allergy Clin Immunol 2008; 121(4):947-954.

[3] Yew Y W, Thyssen J P, Silverberg J I. A systematic review and meta-analysis of the regional and agerelated differences in atopic dermatitis clinical characteristics. J Am Acad Dermatol 2019; 80(2):390-401.

[4] Otsuka A, Nomura T, Rerknimitr P, Seidel JA, Honda T, Kabashima K. The interplay between genetic and environmental factors in the pathogenesis of atopic dermatitis. Immunol Rev 2017; 278(1):246-262.

[5] Eichenfield L F, Ahluwalia J, Waldman A, Borok J, Udkoff J, Boguniewicz M. Current guidelines for the evaluation and management of atopic dermatitis: A comparison of the Joint Task Force Practice Parameter and American Academy of Dermatology guidelines. J Allergy Clin Immunol 2017;139(4S):S49-S57.

[6] Paulsen C E, Armache J P, Gao Y, Cheng Y, Julius D. Structure of the TRPA1 ion channel suggests regulatory mechanisms. Nature 2015; 520(7548):511-517.

[7] Bautista D M, Jordt S E, Nikai T, Tsuruda PR, Read AJ, Poblete J, et al. TRPA1 mediates the inflammatory actions of environmental irritants and proalgesic agents. Cell 2006; 124(6):1269-1282.

[8] Wilson S R, Gerhold K A, Bifolck-Fisher A, Liu Q, Patel KN, Dong X, et al. TRPA1 is required for histamine-independent, Mas-related G protein-coupled receptor-mediated itch. Nat Neurosci 2011; 14(5):595-602.

[9] Gouin O, L'Herondelle K, Lebonvallet N, Le Gall-Ianotto C, Sakka M, Buhé V, et al. TRPV1 and TRPA1 in cutaneous neurogenic and chronic inflammation: pro-inflammatory response induced by their activation and their sensitization. Protein Cell 2017;8(9):644-661.

[10] Kittaka H, Tominaga M. The molecular and cellular mechanisms of itch and the involvement of TRP channels in the peripheral sensory nervous system and skin[J]. Allergol Int, 2017,66(1):22-30.

[11] Liu B, Escalera J, Balakrishna S, et al. TRPA1 controls inflammation and pruritogen responses in allergic contact dermatitis[J]. FASEB J, 2013,27(9):3549-3563.

[12] Kemeny A, Kodji X, Horvath S, Komlódi R, Szőke É, Sándor Z, et al. TRPA1 Acts in a Protective Manner in Imiquimod-Induced Psoriasiform Dermatitis in Mice. J Invest Dermatol 2018;138(8):1774-1784.

[13] Feng J, Yang P, Mack M R, Dryn D, Luo J, Gong X, et al. Sensory TRP channels contribute differentially to skin inflammation and persistent itch. Nat Commun 2017;8(1):980.

[14] Nattkemper L A, Tey H L, Valdes-Rodriguez R, Lee H, Mollanazar NK, Albornoz C, et al. The Genetics of Chronic Itch: Gene Expression in the Skin of Patients with Atopic Dermatitis and Psoriasis with Severe Itch. J Invest Dermatol 2018;138(6):1311-1317.

[15] Kitamura A, Takata R, Aizawa S, Watanabe H, Wada T. A murine model of atopic dermatitis can be generated by painting the dorsal skin with hapten twice 14 days apart. Sci Rep 2018;8(1):5988.

[16] Saarnilehto M, Chapman H, Savinko T, Lindstedt K, Lauerma AI, Koivisto A. Contact sensitizer 2,4dinitrochlorobenzene is a highly potent human TRPA1 agonist. Allergy 2014; 69(10):1424-1427.

[17] Lee JH, Choi CS, Bae IH, Choi JK, Park YH, Park M. A novel, topical, nonsteroidal, TRPV1 antagonist, PAC-14028 cream improves skin barrier function and exerts anti-inflammatory action through modulating epidermal differentiation markers and suppressing Th2 cytokines in atopic dermatitis. J Dermatol Sci 2018. doi: 10.1016/j.jdermsci.2018.04.017.

[18] Dawn A, Papoiu AD, Chan YH, Rapp SR, Rassette N, Yosipovitch G. Itch characteristics in atopic dermatitis: results of a web-based questionnaire. Br J Dermatol 2009;160(3):642-644.

[19] Yosipovitch G, Goon A T, Wee J, Chan YH, Zucker I, Goh CL. Itch characteristics in Chinese patients with atopic dermatitis using a new questionnaire for the assessment of pruritus. Int $\mathrm{J}$ Dermatol 2002;41(4):212-216. 
[20] Chrostowska-Plak D, Reich A, Szepietowski JC. Relationship between itch and psychological status of patients with atopic dermatitis. J Eur Acad Dermatol Venereol 2013;27(2):e239-e242.

[21] Tóth BI, Oláh A, Szöllősi AG, Bíró T. TRP channels in the skin. Br J Pharmacol 2014;171(10):2568-2581.

[22] Wilson S R, Gerhold K A, Bifolck-Fisher A, Liu Q, Patel KN, Dong X, et al. TRPA1 is required for histamine-independent, Mas-related G protein-coupled receptor-mediated itch. Nat Neurosci 2011;14(5):595602.

[23] Wilson S R, The L, Batia L M, Beattie K, Katibah GE, McClain SP, et al. The epithelial cell-derived atopic dermatitis cytokine TSLP activates neurons to induce itch. Cell 2013;155(2):285-295.

[24] Cevikbas F, Wang X, Akiyama T, Kempkes C, Savinko T, Antal A, et al. A sensory neuron-expressed IL-31 receptor mediates T helper cell-dependent itch: Involvement of TRPV1 and TRPA1. J Allergy Clin Immunol 2014;133(2):448-460.

[25] Oh M H, Oh S Y, Lu J, Lou H, Myers AC, Zhu Z, et al. TRPA1-dependent pruritus in IL-13-induced chronic atopic dermatitis. J Immunol 2013;191(11):5371-5382.

[26] Modena B D, Dazy K, White AA. Emerging concepts: mast cell involvement in allergic diseases. Transl Res 2016;174:98-121.

[27] Hox V, Vanoirbeek JA, Alpizar YA, Voedisch S, Callebaut I, Bobic S, et al. Crucial role of transient receptor potential ankyrin 1 and mast cells in induction of nonallergic airway hyperreactivity in mice. Am J Respir Crit Care Med 2013;187(5):486-493.

[28] Kang J, Ding Y, Li B, Liu H, Yang X, Chen M. TRPA1 mediated aggravation of allergic contact dermatitis induced by DINP and regulated by NF-kappaB activation. Sci Rep 2017;7:43586.

[29] Kasraie S, Werfel T. Role of macrophages in the pathogenesis of atopic dermatitis. Mediators Inflamm 2013;2013:942375.

[30] Sica A, Mantovani A. Macrophage plasticity and polarization: in vivo veritas. J Clin Invest 2012;122(3):787-795.

[31] Nakai K, He Y Y, Nishiyama F, Naruse F, Haba R, Kushida Y, et al. IL-17A induces heterogeneous macrophages, and it does not alter the effects of lipopolysaccharides on macrophage activation in the skin of mice. Sci Rep 2017;7(1):12473.

[32] Chao LK, Hua KF, Hsu HY, Cheng SS, Lin IF, Chen CJ, et al. Cinnamaldehyde inhibits proinflammatory cytokines secretion from monocytes/macrophages through suppression of intracellular signaling. Food Chem Toxicol 2008;46(1):220-231.

[33] Usui-Kusumoto K, Iwanishi H, Ichikawa K, Okada Y, Sumioka T, Miyajima M, et al. Suppression of neovascularization in corneal stroma in a TRPA1-null mouse. Exp Eye Res 2019;181:90-97.

[34] Wang Z, Xu Y, Wang M, Ye J, Liu J, Jiang H, et al. TRPA1 inhibition ameliorates pressure overloadinduced cardiac hypertrophy and fibrosis in mice. EBioMedicine 2018;36:54-62.

[35] Moyle M, Cevikbas F, Harden JL, Guttman-Yassky E. Understanding the Immune Landscape in Atopic Dermatitis: The Era of Biologics and Emerging Therapeutic Approaches. Exp Dermatol 2019;28(7):756-768. 